SAND94-8535

UC-406

Unlimited Release

Printed March 1994

\title{
The Role of Variation, Error, and Complexity in Manufacturing Defects*
}

\author{
C. Martin Hinckley \\ Project and Administration Department \\ Sandia National Laboratories / California
}

\author{
Professor Philip Barkan \\ Stanford University \\ Stanford, CA 94305-4021
}

\begin{abstract}
Variation in component properties and dimensions is a widely recognized factor in product defects which can be quantified and controlled by Statistical Process Control methodologies. Our studies have shown, however, that traditional statistical methods are ineffective in characterizing and controlling defects caused by error. The distinction between error and variation becomes increasingly important as the target defect rates approach extremely low values. Motorola data substantiates our thesis that defect rates in the range of several parts per million can only be achieved when traditional methods for controlling variation are combined with methods that specifically focus on eliminating defects due to error.
\end{abstract}

Complexity in the product design, manufacturing processes, or assembly increases the likelihood of defects due to both variation and error. Thus complexity is also a root cause of defects. Until now, the absence of a sound correlation between defects and complexity has obscured the importance of this relationship.

We have shown that assembly complexity can be quantified using Design for Assembly (DFA) analysis. High levels of correlation have been found between our complexity measures and defect data covering tens of millions of assembly operations in two widely different industries. The availability of an easily determined measure of complexity, combined with these correlations, permits rapid estimation of the relative defect rates for alternate design concepts. This should prove to be a powerful tool since it can guide design improvement at an early stage when concepts are most readily modified.

* Based on a journal article submitted to the Journal of Quality Technology

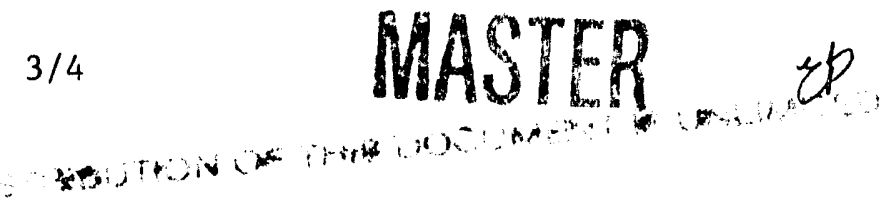




\section{Table of Contents}

I. Introduction. 9

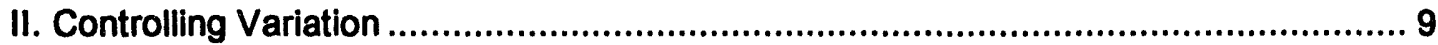

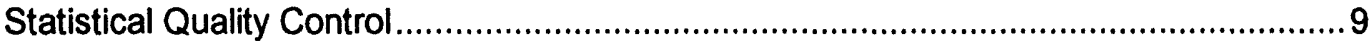

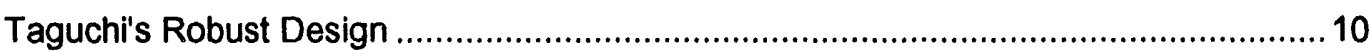

Motorola's 6 Sigma and the Process Capability Index ................................................ 10

Process Capability - An Insufficient Measure for Predicting Defects .........................11

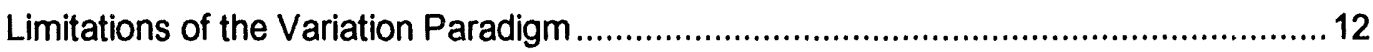

III. Error - a Distinctly Different Defect Source than Variation ....................................13

Error Probabilities Are Not Predicted by Variation Models ...................................... 14

Probability-The Only Universal Method for Describing Error and Variation .............. 14

Error-A Critical Factor in Defect Creation ............................................................ 14

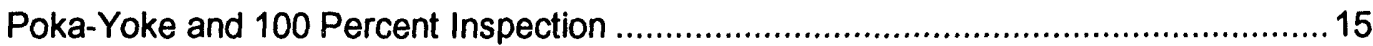

Evidence Supporting the Distinction Between Variation and Error ............................... 16

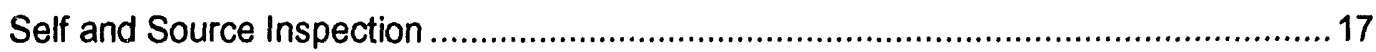

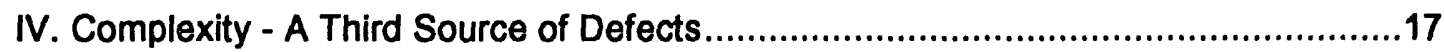



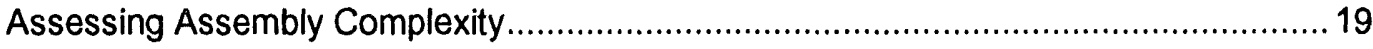

Assembly Complexity - A Key Factor in Conformance Quality .................................... 19



Testing the Relationship Between Defects and Assembly Complexity ..........................20

Assessing the Level of Quality Control for Each Organization ......................................22

Comparison of Product Concepts - An Illustrated Example...........................................22

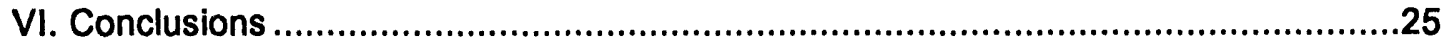




\section{List of Illustrations}

1 Population and sample from Ishikawa ..................................................... 10

2 The probability distribution functions for a hole and cylinder diameter ............... 11

3 Part proposed by Olivera (1988) for the study of variation.............................. 13

4 The relationship between complexity, error, and variation defects ...................... 18

5 Defect rate versus manual Assembly Efficiency by Brannan (1991) ..................... 19

6 Defects per unit versus assembly complexity for two manufacturers.................. 21

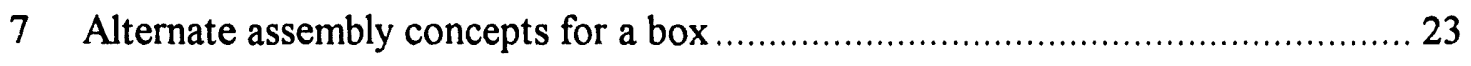

8 Predicted assembly time versus number of assembly operations .......................... 24

\section{List of Tables}

1 Estimated defects per unit for four product concepts ................................ 23 


\section{Introduction}

Buzzell (1987) et al proclaimed that "Quality is King," affirming its dominant role in market share and Return on Investment. The performance of Japanese products in the marketplace reinforces this conclusion (Womack et. al. (1990)). Our focus is motivated by tremendous pressure to improve conformance quality, measured by the manufacturing defect rate, to previously unimaginable limits.

Illustrating the required level of performance, Toyota asserted in 1990 that their North American suppliers had defect rates that were two orders of magnitude higher than their Japanese suppliers (Sanger (1990)). Similarly, the goal of Motorola's 6 sigma (Harry et. al. (1988)) program has been to reduce defects by roughly 3 orders of magnitude. In both cases the new target for defect levels is in the range of 1 to 10 parts per million (ppm). By contrast, defects in the range of 2,000 to $20,000 \mathrm{ppm}$ are perceived as normal using traditional Statistical Quality Control (SQC) (Ishikawa (1990)).

The consequences of this trend are profound, pointing to the need for a new rigor in understanding defects as an essential element for improvement. It is not enough to confront defect issues on the factory floor. A means of addressing potential defect sources early in the design process is essential, requiring a comprehensive approach.

Several concepts and methods have evolved which have the purpose of reducing defects. The focus of these methods often leads to strategies for defect reduction that are limited, particularly when the goal is to achieve defect rates below $10 \mathrm{ppm}$. We will first critically review the sources of product defects and the traditional methodologies that have been developed to address the defect sources.

\section{Controlling Variation}

\section{Statistical Quality Control}

Statistical Quality Control (SQC) was one of the earliest quality improvement methods to be developed. It has become the backbone of several other techniques. It is based on the principle that variation can be observed in all processes and that such variation can be described statistically. Building upon this concept, sampling has been used to characterize process dispersion and control processes. Figure 1, based on the work of Ishikawa (1990), illustrates a sampling, measurement, and feedback cycle which is the basis for this method. 




Figure 1. Population and Sample from Ishikawa (1990).

This traditional Quality Control method has two major shortcomings:

1. Defects or deviations are detected after they are created, as Figure 1 shows. The number of defects produced is very sensitive to the response time of the feedback cycle, which can involve many delays when the point of detection is downstream from the defect source.

2. Traditional Quality Control focuses exclusively on the production process, accepting the design as given. Since many defects are attributable to design, a focus on eliminating defects in the production process misses many important opportunities for defect reduction.

In one sense, the two major shortcomings of traditional quality control are related since they both center on a downstream process control. This represents a serious limitation since defect discovery is generally too late for maximum effectiveness.

\section{Taguchi's Robust Design}

Taguchi's (Ross 1988) robust design represents an important advance in this perspective. This method addresses defect problems during the design/development phase. This method considers defect issues relative to the product design as well as the production process. Taguchi robust design seeks defect reduction (and improved reliability) by reducing sensitivity to variation. Largely an experimental procedure which comes late in the design process, its power is limited by its very limited application in the earliest phases of design.

\section{Motorola's 6 Sigma and the Process Capability Index}

Motorola's 6 sigma (Harry et al (1988)) seeks to control defects caused by variation through assuring that design requirements have been correctly established in the design phase and that the production process capability matches these requirements. Like the Taguchi method it can influence the design as well as the production process. An important measure used by Motorola's 6 sigma method to identify appropriate production processes is the Process Capability Index, $C_{p}$ (Harry et al (1988)). The Process Capability Index provides a measure of how well variation is controlled relative to tolerance limits. For bilateral tolerances, this index (which has also been called the "standard capability ratio" Gryna (1988)) is defined as: 


$$
C_{p}=\frac{|U S L-L S L|}{6 \cdot \sigma}=\frac{\text { tolerance width }}{\text { process capability }}
$$

$$
\begin{aligned}
\text { Where, USL } & =\text { Upper Specification Limit } \\
\text { LSL } & =\text { Lower Specification Limit } \\
\sigma & =\text { the Standard Deviation of the production process }
\end{aligned}
$$

A related index $\left(\mathrm{C}_{\mathrm{p}_{\mathrm{k}}}\right)$ (Harry et al (1988)) addresses "shifts and drifts" in the process mean. Motorola has based the 6 sigma method upon the likelihood of a mean shift that is equal to 1.5 standard deviations.

\section{Process Capability - An Insufficient Measure for Predicting Defects}

The $\mathrm{C}_{\mathrm{p}}$ and $\mathrm{C}_{\mathrm{p}_{\mathrm{k}}}$ indices can be used to predict how frequently the outcome of a process will exceed specified limits. However, the probability of a defect is significantly different from the probability of exceeding specification limits. By way of illustration, the distribution for two manufacturing processes, a cylinder turning operation and hole forming operation, are shown in Figure 2. For the normal distributions given in Figure 2, the probability that a cylinder will exceed the Upper Specification Limit is 0.0013 . Similarly, the probability that a hole has a diameter smaller than its Lower Specification Limit is 0.0013 . However, the probability of encountering interference in a random assembly of the cylinder and hole from Figure 2 must be determined by examining the joint probability of having a large cylinder and small hole that interfere. The probability of assembly interference in this case is only $2.13 \mathrm{e}-5$.



Figure 2. The probability distribution functions (PDF) for a cylinder turning and hole forming operation based on an ANSI standard class LC4 clearance locational fit (Oberg et. al. (1984)). Note the logarithmic vertical scale. The vertical lines indicate assumed Lower Specification Limits (LSL) and the Upper Specification Limits (USL). The cylinder has a mean diameter of 0.749 inches with a standard deviation of 0.00033 inches. The hole drilling distribution is based on a mean of 0.75175 inches with a standard deviation of 0.000583 . 
This type of analysis reveals that there is a high probability with random assembly that an oversized cylinder will not cause interference during assembly. As this case illustrates, the probability of a interference or a defect caused by variation is generally lower than the probability of exceeding the specification limits (assuming the limits have been set correctly). However, defect rates based on joint probability distributions are extremely sensitive to the accurate characterization of the tails of the distribution.

Accurate assessment of joint probabilities and the correct assignment of specification limits becomes increasingly difficult as the number of variables in a study grows. Frequently, simplifying assumptions are used to make analysis of variation practical. For example, a Root Sum of Squares (RSS) (Harry et al (1988)) method is commonly used in conjunction with one dimensional analysis (Research (1988)) to define tolerance specifications. However, more sophisticated three dimensional treatments of tolerance variations that depend on Monte Carlo simulations have shown that the RSS method is inaccurate for complex problems (Heggem (1992), Held (1993)). We have shown that interference probabilities can be underestimated by orders of magnitude when complex interfaces are evaluated as one dimensional rather than three dimensional problems (Hinckley (1993)). As a consequence, the Process Capability Index is generally an inaccurate predictor of defect rates even though it is a useful tool in controlling variation.

Based on the drilling variation illustrated in Figure 2, we note that the predicted probability of inadvertently forgetting to drill a hole (diameter $=0.0^{\prime \prime}$ ) is virtually zero since this outcome differs from the mean diameter by 1280 standard deviations.

\section{Limitations of the Variation Paradigm}

As typically applied, each of the methods that focus on variation are based on three fundamental assumptions:

1) Sampling can be used to characterize all significant sources of variation,

2) All defects are a result of variation, and

3) It is generally assumed that variation follows a Normal or Gaussian distribution. Based on these assumptions, in practice sample sizes are generally small and "odd-ball" readings are discarded or averaged in a manner that dilutes their significance. Such practices, while convenient, effectively preclude an accurate assessment of the "tails" of the distribution. Since defects arise from the tails of the distributions, the assumption of a normal distribution can seriously underestimate the incidence of defects. Our work has challenged each of the underlying assumptions that have led to current practices. Understanding the nature of rare events, particularly those beyond three standard deviations from the mean, and the limitations of statistical methods is particularly important when the goal is to achieve extremely low defect rates below $10 \mathrm{ppm}$. 


\section{Error - a Distinctly Different Defect Source than Variation}

Although variation is a useful way of describing the cause of many defects, it does not describe the cause of all defects. To illustrate, in the assembly of the simple box shown in Figure 3, an operator may occasionally forget to install a screw. In the assembly, each screw is either missing or present, a condition that can only be described in terms of probability rather than variation. Omitting a screw is an error rather than a variation. We define error as the execution of a prohibited action, or the failure to perform a required action, or the misinterpretation of information essential for the correct execution of an action.



Figure 3. Part proposed by Olivera (1988) for the study of defects resulting from variation. Omitting a screw, an error, illustrates a type of defln the fabrication and assembly of the box illustrated in Figure 3, there are several possible types of omission errors that can be identified. For example, 1) an operator may install a screw, but forget to tighten it, 2) occasionally an operator may forget to drill one of the clearance holes in the lid, 3) or the operator may forget to tap one of the holes that accepts a screw. Collectively, with the error of omitting a screw, four types of omission errors have been described for each threaded connector in this assembly.

In a clear departure from the predictions based on variation, Rook (1962) found, in a study of over 23,000 production defects, that the probability of omitting an operation and not detecting the omission using traditional production practice was approximately 0.00003 . In other words, roughly one operation in 33,000 will be omitted without detection, resulting in a defect rate of $30 \mathrm{ppm}$. Given two threaded fasteners and four types of omission errors per fastening feature, the cumulative defect rate resulting from omission errors for the simple box illustrated in Figure 3 would be approximately $240 \mathrm{ppm}$ (60 ppm per part). This exceeds the defect rate goal of $1-10 \mathrm{ppm}$ by roughly an order of magnitude. 


\section{Error Probabilities Are Not Predicted by Variation Models}

The distributions describing process variation are useless in predicting the frequency of errors. For example, variation in screw torque can only be measured for screws that are installed and tightened. Thus, the probability that the screw is omitted or that an operator forgets to tighten the screw is not predicted by the distribution of torque. Similarly, the distribution of clearance hole diameters can not be used to estimate the frequency of omitting a drilling operation since the observed distribution can only be based on the measurement of holes that have been drilled. Thus, the outcome of errors exceeds the frequency and magnitude predicted by methods based on variation.

Because errors occur rarely in production, traditional sampling and statistical methods are not useful in estimating their frequency. To illustrate, for a ninety percent confidence of observing just fifteen screw omission errors while sampling one operation in a hundred, we would have to perform over a hundred million operations! Thus, we cannot depend upon sampling or statistical methods to predict how frequently errors are occurring. More importantly, statistical methods are not effective in eliminating defects caused by errors. Errors require different methods of control than variation, and consequently must be treated as a separate source of defects.

We have found support for this assessment in data drawn from 23 independently observed studies of human performance which were obtained from research and production environments (Hinckley (1993)). We have consistently rejected the normal distribution at the highest levels of significance in these cases where it has been traditionally assumed for decades, demonstrating that functions derived from Pareto's law provide a clearly superior model of the data in every case. Since Pareto's law deviates from the conditions of the Central Limit Theorem, this law provides a theoretical explanation for the observation that traditional statistical methods consistently underestimated the frequency and magnitude of rare events for these data, revealing a critical limitation of statistical methods in predicting or controlling defect rates.

\section{Probability-The Only Universal Method for Describing Error and Variation}

We can convert every distribution describing variation to probabilities. For example, we can extrapolate from a given distribution to predict the probability of exceeding specification limits, or the probability of interference. However, we cannot convert every probability to a distribution.

As we have already shown, error can only be effectively described in terms of probability. Errors either occur or they do not. A part is either present in the product or missing. Consequently, the only universal method of describing both error and variation is probability.

\section{Error-A Critical Factor in Defect Creation}

Although some writers have alluded to a difference between mistakes or error, and variation (Lafferty (1992)), this distinction has not been accurately described in the 
literature. This is probably due to the fact that it is virtually impossible to accurately assess rare events using sampling methods. As shown by our example, the assumption that variation alone can characterize all defects is flawed and often prevents identification of major defect sources.

The relative control of variation and error differs for each organization. We have developed a technique which aids in assessing the relative strengths and weaknesses in controlling error and variation based on each organizations defect data and quality control philosophy. However, a discussion of this method is beyond the scope of this paper.

Errors of omission represent only one type of error that can occur. The following types of errors were listed "in order of importance" in a recent book edited by the Nikkan Kogyo Shimbun, Ltd. (1988):

\section{Omitted processing}

2. Processing errors

3. Errors sctting up workpieces

4. Missing parts

5. Wrong parts
6. Processing the wrong piece

\section{Misoperation}

8. Adjustment error

9. Equipment not set up properly

10. Tools and jigs improperly prepared

While each of these errors are individually rare occurrences, collectively they can have a significant impact on conformance quality. Harris et al (1969) concluded that 80 percent of the defects in complex systems could be attributed to error. In an examination of 23,000 production defects, Rook (1962) found that 82 percent of all defects were caused by human errors. Voegtlen (1988) reported that 60 percent of product failures could be traced to workmanship defects. In our review of data for front end automotive headlamps by a major manufacturer we also observed that more the 70 percent of 6,600 observed defects were caused by assembly or handling errors (Hinckley (1993)). In addition, NASA (Associated Press (1993)) reported that most space shuttle mishaps occurring since October 1990 are the result of human error. All of these studies point to human error as a key source of defects and failures.

\section{Poka-Yoke and 100 Percent Inspection}

Shingo (1986) makes an important distinction between error and product defects. While errors are inevitable, defects are not. He has shown that, in many cases, simple methods can provide early error detection that assure correction before a defect is passed to the next stage in the production process. He stated:

"We should recognize that people are, after all, only human and as such, they will, on rare occasions, inadvertently forget things. It is more effective to incorporate a checklist-i.e., a poka-yoke-into the operation so that if a worker forgets something, the device will signal that 
fact, thereby preventing defects from occurring. This, I think, it the quickest road leading to attainment of zero defects." (italics added) (Shingo (1986))

This is consistent with observations made by Rasmussen (1985), who concluded that the frequency of errors derived from incident reports (such as defects) is dependent on the opportunity for people to detect and correct the errors immediately. No amount of vigilance or training will assure that unintentional errors will be recognized. A core concept is that poka-yoke, in combination with 100 percent inspection, can catch virtually every error.

Using these techniques, a washing machine drainpipe assembly line processing 30,000 units a month involving 23 workers achieved zero defects for six consecutive months (Shingo (1986)). This level of performance is orders of magnitude better than the lowest estimates of human error rates per Rook (1965). By using poka-yoke to detect and correct error, defect probabilities can be less than error probabilities. Consequently, defects are related more to the level of quality control than to the frequency of errors.

As illustrated, centering attention on error prevention and intervention is more productive than prediction of error rates for production problems. Poka-yoke can be incorporated in the product to assure that a part cannot be assembled in the wrong position or orientation (Tsuda (1993)). In some instances it is possible to design the product so that assembly cannot proceed if a part is missing or incorrectly positioned. In this sense, poka-yoke is an important design tool. In practice, however, poka-yoke is not a full solution because it is largely limited to specific accessible process steps and the design is accepted as given.

\section{Evidence Supporting the Distinction Between Variation and Error}

The distinction between error and variation is critical in achieving the lowest defect rates demanded by the marketplace. Using traditional Statistical Quality Control (SQC), defect rates in the range of $2000 \mathrm{ppm}$ can be achieved. Based on normally distributed variation and Motorola's 6 sigma philosophy, defect rates can theoretically be reduced to the 3.4 ppm through the control of variation. However, we estimated that the 6 sigma concepts would only reduce defect rates by roughly a factor of two because this method does not address errors. After five years of aggressively applying the 6 sigma philosophy, data supplied by Motorola (Gebala (1992)) showed that their average defect rate on several products was more than two orders of magnitude greater than the 6 sigma goal of 3.4 ppm, a result consistent with our estimates.

Some may argue that Motorola has simply not achieved their 6 sigma goal. However, evidence that this is not the case can be obtained from information recently published by Motorola in September of 1993. Smith (1993) stated,

"Motorola elected to enter this market (electronic ballast) and set a quality goal of 6 sigma for initial delivery. This required a very strict TDU (total number of defects per unit) budget. But it became evident early in the project that achieving a $\mathrm{C}_{\mathrm{p}}$ greater than 2 would go only part 
of the way. Mistake-proofing the design would also be required...Mistake-proofing the design is an essential factor in achieving the TDU goal. The design team is forced to investigate any opportunities for errors during manufacture and assembly, and to eliminate them." (italics added) (Smith (1993))

Motorola's own experience clearly demonstrates that mistake-proofing (the English translation of poka-yoke per Shingo (1986)) must be applied in conjunction with control of variation to achieve the lowest defect rates. With the addition of error control, Motorola was able to achieve defect rates in the tens of parts per million.

\section{Self and Source Inspection}

Shingo has also introduced several quality concepts that overcome some of the limitations of other methods. Self inspection, and source inspection (Shingo (1986)) have the goal of detecting and eliminating defects at their production source. Self inspection has the objective of detecting defects as close to the point of gencration as possible to reduce or eliminate delays in the feedback. By gaging tools, materials and activities upstream of the process, it is possible to eliminate many defects before they are created using source inspection. However, these techniques, like Statistical Quality Control (SQC), do not address design as an important cause of defects.

\section{Complexity - A Third Source of Defects}

There is another source of defects which we refer to as "complexity." Returning to the box example illustrated in Figure 3, if the lid could be attached with one screw instead of two, several opportunities for error would be eliminated and the number of features contributing to variation deferts would be reduced. A snap fit lid could further reduce the probability of error and variation defects. Thus, many defects resulting from either error or variation can be avoided through reducing the complexity of the product design.

This view is consistent with numerous studies which have identified task complexity or difficulty as a factor increasing the probability of errors (Swain et al (1983), Meister (1961), Park (1987), Card et al (1978), Jerison (1963), Harris (1966), MacKenzie et al (1991), Gatchell (1979), Stalk et al (1990)). Although this relationship is widely recognized, Rasmussen (1981) stated that it has not been quantified in general terms. Our own work has shown that complexity also increases defects resulting from variation as well as errors (Hinckley (1993)).

The relationship between error, variation and complexity is illustrated in Figure 4 . Since both defects due to error and variation are rare events, we can treat them as independent defect sources. Neither error nor variation are, by themselves, a complete description of the cause of defects. However, complexity influences the likelihood of defects in a product resulting from either variation or error. Consequently, the link between complexity and defects leads to a more global or general model of defects than either 
error or variation considered independently. In the remainder of the paper we will focus on the broader relationship between defects and complexity.

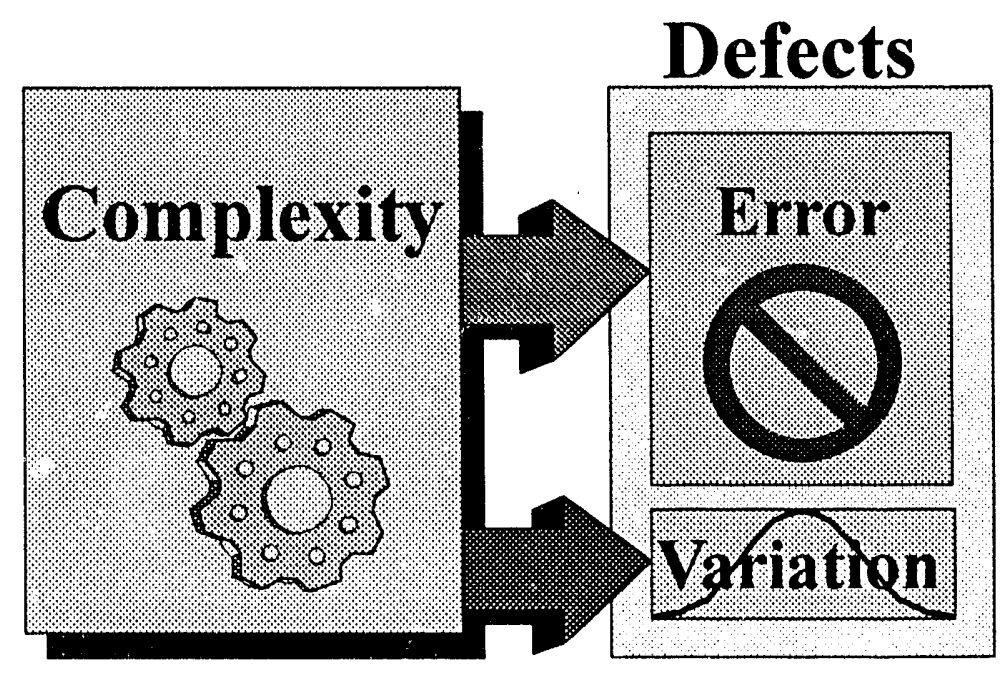

Figure 4. The Relationship between complexity, error, variation and defects. Since excessive variation and error are both rare events, they can be treated as independent defect sources which, by themselves, are not complete models of defects. Complexity increases both errors and defects resulting from variation.

Some earlier efforts have been made to test on a broad basis the relationship between product complexity and defect rates (Womack et al (1990), Ekings (1988)). These efforts have led to weak correlations which we attribute to two factors: 1) oversimplified measures of complexity, and 2) differences in quality control between organizations.

\section{Quantifying Complexity}

At the present, there are only a few relative and no absolute measures of complexity. Control of product complexity, a major source of defects, has thus far been hampered by this lack of a quantifiable basis of measurement.

We introduce a general perspective on complexity, proposing that a valid measure of this characteristic must have two essential elements:

a) a quantity measure - identifying the number of elements which contribute to complexity

b) a difficulty measure - a relative measure of the difficulty in generating or executing each of the elements.

A common weakness in efforts to understand the role of complexity has been the singular focus on the number of elements. It is gererally easy to count the number of elements contributing to complexity, such as the number of assembly operations in a product. However, assessing the relative difficulty of the elements is challenging. At the present, there are no generally accepted methods for comparing part complexity. However, 
Design for Assembly (DFA) methods have been developed in the last decade which we believe have the potential for assessing assembly complexity.

\section{Assessing Assembly Complexity}

Design for Assembly (DFA) methodologies were developed with the objective of helping designers identify opportunities for making assembly easier. In every method, the number of parts, and assembly operations, quantity measures of complexity, are counted.

Virtually, all of these methodologies also translate handling, insertion, and securing actions into an estimate of the nominal time needed to perform an assembly process. In general, for every factor that increases the difficulty of the action or the complexity of the assembly interface, there is an increase in the predicted time for execution. The assembly time per operation provides an approximate relative comparison of the difficulty or complexity of the dissimilar assembly activities. Thus, the Design for Assembly (DFA) methods address both quantity and difficulty measures of complexity.

\section{Assembly Complexity - A Key Factor in Conformance Quality}

In late 1990 Motorola published a report (Brannan (1990)) which showed that the number of defects per million parts decreased dramatically with increases in the "manual assembly efficiency." Manual assembly efficiency is an arbitrary measure used in the Boothroyd Dewhurst ${ }^{\circledR}$ (1985) Design for Assembly (DFA) method. Motorola's data illustrating the relationship between defects and assembly efficiency is shown in Figure 5.

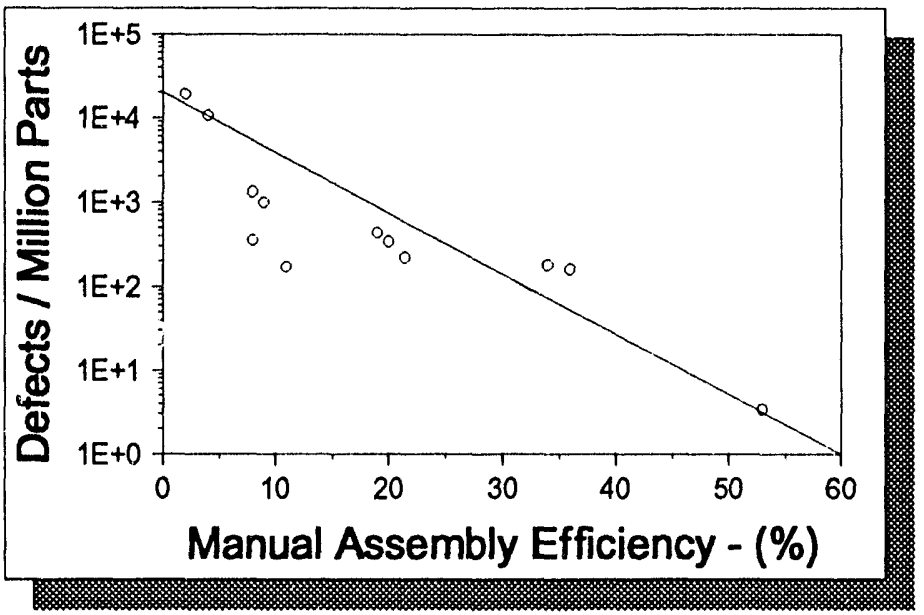

Figure 5. Observed Defects per Million Parts versus the Manual Assembly Efficiency published by Motorola (Brannan (1991)). The line drawn by Motorola is not a curve fit.

To explain the relationship in Figure 5, consider that the number of assembly operations and the average time required to perform each operation generally decreases as the assembly efficiency increases. Assuming a constant probability of human error per unit time, the defect rate should increase as the assembly time per operation or complexity 
ircreases. Thus, the relationship between defects and assembly efficiency observed by Motorola is intuitively sound. Motorola did not attempt to explain this relationship.

The data provided by Motorola piqued our interest because it suggested that there may be a quantifiable link between a criteria describing assembly complexity and product defects. Such a correlation could be used to evaluate the conformance quality potential of product concepts before tolerance studies are even initiated. In addition, a model based on such a relationship could provide important insights useful in defining quality improvement strategies (Barkan (1993))

\section{A New Model of Conformance Quality}

\section{Testing the Relationship Between Defects and Assembly Complexity}

To test the relationship between assembly complexity measured using DFA and defect rates, extensive data were obtained from Motorola (Gebala (1992)) and a disk drive manufacturer. For each data set, there are many possible ways of comparing defect rates to complexity measures. For example, defect rates can be expressed in terms of defects per operation, defects per part, or defects per assembly, and assembly complexity could be described in terms of total assembly time, average time per assembly operation, or average assembly time per part. In our study, more than 50 univariate and multivariate comparisons of defect rates and complexity measures were made for the data supplied by each manufacturer.

While defects did not have any consistent correlation with part count, the defect-tocomplexity relationship illustrated in Figure 6 was found to provide the highest correlation for data of both manufacturers. This relationship links the Defects per Unit (DPU) to a function of total DFA predicted assembly time (TM) and the number of assembly operations $\left(\mathrm{N}_{\mathrm{a}}\right)$. The relationship is remarkable not only for the high correlations (correlation coefficients $(r)>0.95$ ) but also for the observed consistency for data from two different manufacturers. Each point in the figure represents a different product or subassembly. Collectively the data reflects defect rates involving tens of millions of parts and assembly operations. 


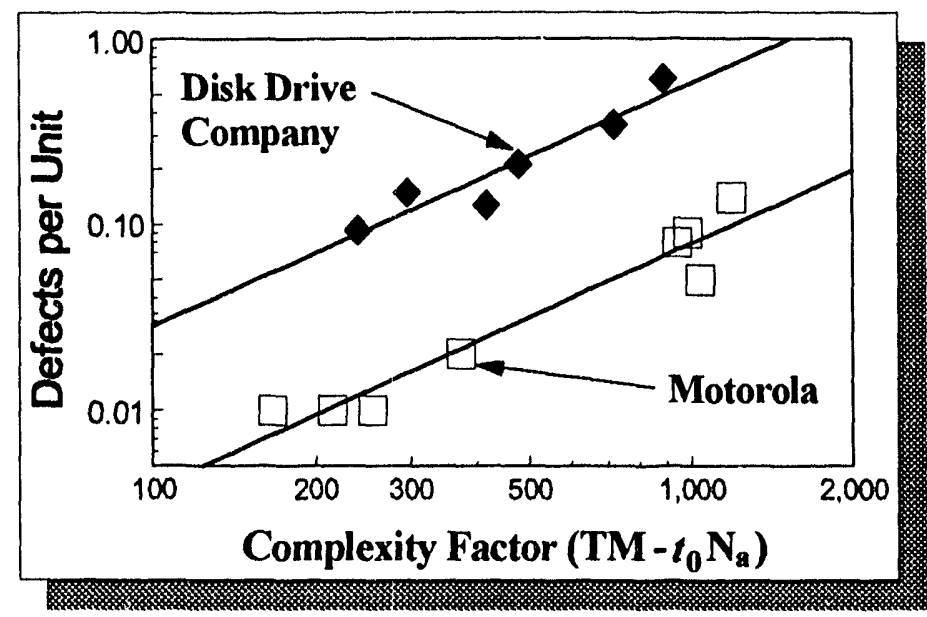

Figure 6. Defects per unit versus assembly complexity for Motorola (Gebala (1992)) and disk drive manufacturer. The solid lines represent power curve fits to the data. Each point represents a different product or subassembly.

The equation defining the model of defects per unit (DPU) versus assembly complexity is as follows:

$$
\mathrm{DPU}=\frac{\left(\mathrm{TM}-\boldsymbol{t}_{0} \cdot \mathrm{N}_{\mathrm{a}}\right)^{\bar{k}}}{\mathrm{c}_{3}}
$$

$t_{0}, \bar{k}$, and $\mathrm{c}_{3}$, are three constants. The constant $\boldsymbol{t}_{0}$ represents a threshold assembly time for the simplest assembly operation. The constant $\bar{k}$ is the slope of the data in Figure 6 , which is approximately equal to 1.3 for both sets of data, and the constant $c_{3}$ is related to the vertical position of the data in the same figure.

Equation 1 is the core of a new model of production defects that is more general or global in nature. This comprehensive model is more fundamentally sound and better validated by correlation with production performance than any other current approach to predicting defect rates. We are not aware of any methods or studies, for example, which have combined all variations in a product to predict general defect rates in a manner that has been correlated with production performance spanning a broad range of product complexity.

The relationships illustrated in Figure 6 clearly show that complexity plays a dominant role in product defects. The differences in the two sets of data also demonstrates that large differences in level of quality control can exist among manufacturers as we had anticipated, explaining why poor correlations between defects and complexity have been observed in previous industry-wide studies. Figure 6 also demonstrates that the relative level of quality control can be easily compared, even for companies producing dissimilar products of different complexity. This promises to be particularly helpful in benchmarking studies. 


\section{Assessing the Level of Quality Control for Each Organization}

To assess the global level of quality control for each organization, in the ideal case Design for Assembly (DFA) data and defect data would be collected for a variety of products currently in production which span a range of complexity for each company. The values of the constants can then be determined by curve fitting as illustrated for two manufacturers in Figure 6. However, this is not always possible for several reasons:

1. Products may be too complex to permit economical DFA evaluations,

2. Lack of cooperation or support prevents collection of defect and DFA data, or

3. Products are so similar in complexity that correlations would not be meaningful. In these cases, if the defect rate and Design for Assembly (DFA) analysis for one existing product can be obtained the relationship between defects and complexity can be estimated by assuming $\bar{k}=1.3$ and $t_{0}=2 \mathrm{sec} /$ operation in the following equation:

$$
c_{3}=\frac{\left(T M-2 \cdot N_{a}\right)^{1.3}}{D P U}
$$

For example, given 500 defects in every million boxes illustrated in Figure $1(\mathrm{TM}=22.5$ $\mathrm{sec}$ and 4 assembly operations), the value of $c_{3}$ could be determined from Equation 2 to be approximately $64,700 \mathrm{sec}^{1.3} \cdot$ units/defect.

\section{Comparison of Product Concepts - An lllustrated Example}

Even where data is limited, one of the most important benefits of this study is that it leads to a method of comparing the relative potential defect rates of product concepts, given a constant level of quality control. This method of comparing concept defect rates can be applied at the earliest stages of concept development before detailed design is begun.

The techniques for comparing product concepts will be illustrated by examining assembly options for the box described by Olivera (1988) as illustrated in Figure 3. The original design and proposed alternate assembly concepts are shown in Figure 7. For each of the proposed assembly concepts, the DFA assembly time and number of assembly operations were determined. These values can be substituted into Equation 1 to estimate the defect rates for a constant level of quality control. The results of this simple analysis, which were completed in about a half an hour, are listed in Table 1. The comparison of the potential defect rates can generally be completed in a fraction of the time required to perform a detailed tolerance analysis of a single concept with predictions that more accurately reflect the defect rates that are likely to be observed in production. 

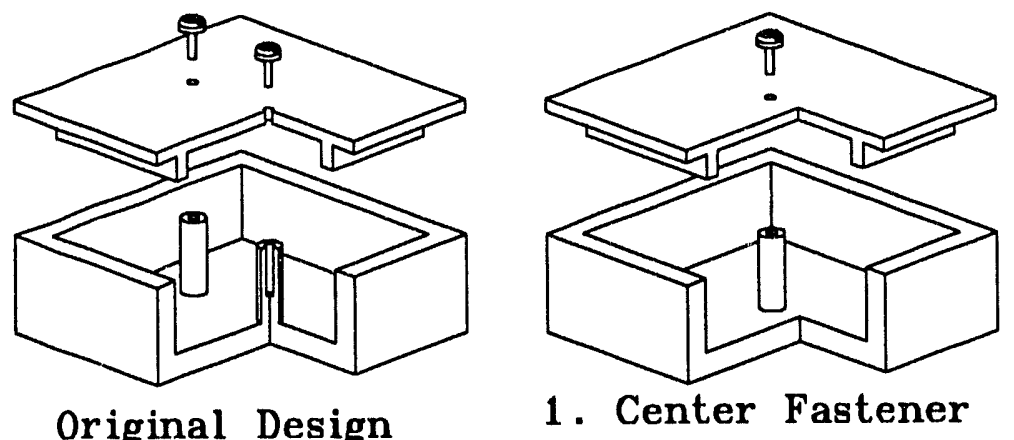

1. Center Fastener
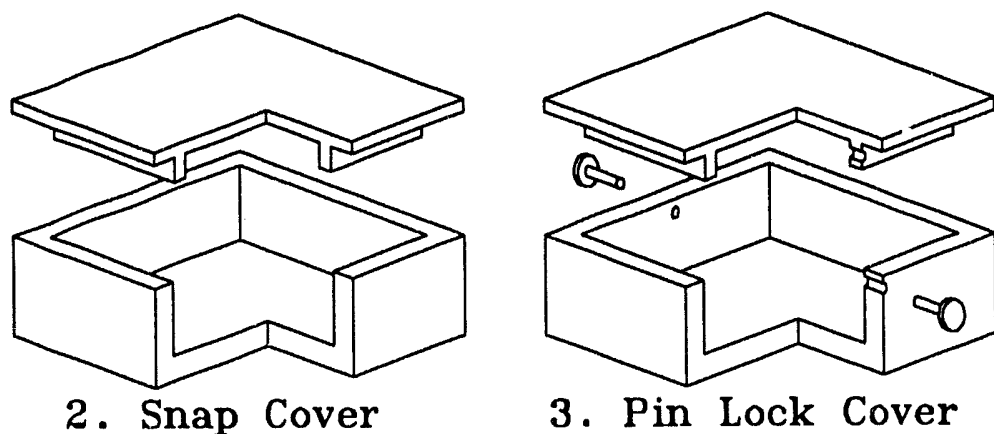

Figure 7. Alternate assembly concepts for a box. The original concept was proposed by Olivera (1988).

Table 1. Estimated Defects per Unit (DPU) for four product concepts illustrated in Figure 7.

Calculations are based on Equation 1 using $\bar{k}=1.3, c_{3}=64,700, t_{0}=2 \mathrm{sec} / \mathrm{op}$. Note, 1.6 seconds has been added to Concept 3 for assembly operations which are not "top down."

\begin{tabular}{|c|c|c|c|c|}
\cline { 2 - 5 } \multicolumn{1}{c|}{} & $\begin{array}{c}\text { Original } \\
\text { Concept } \\
\text { (2 Screws) }\end{array}$ & $\begin{array}{c}\text { Concept 1 } \\
\text { Centered } \\
\text { Screw }\end{array}$ & $\begin{array}{c}\text { Concept 2 } \\
\text { Snap Lid }\end{array}$ & $\begin{array}{c}\text { Concept 3 } \\
\text { Lock Pin }\end{array}$ \\
\hline $\begin{array}{c}\text { DFA Ass'y Time (TM-sec) } \\
\text { Number of Operations (Na) }\end{array}$ & 22.5 & 13.8 & 7 & 16.1 \\
Estimated DPU & 4 & 3 & 2 & 4 \\
\hline \hline Conformance Quality Rank & 4 & 2 & 1 & 3 \\
\hline
\end{tabular}

From the results of this analysis, the snap fit lid will have a defect rate roughly seven times lower than the original product with two screws. Eliminating one screw, or using lock pins will reduce the defect rate by about a factor of two relative to the baseline design. In the absence of other constraints, the snap fit lid would be selected, however, there are often many additional requirements which may favor a product concept having a potential defect rate higher than the minimum alternative. The method presented here simply allows consideration of the potential defect rate as one of the factors to be considered in selecting among design alternatives. 
The difference in potential defect rates among alternative concepts can also be illustrated graphically. Equation 1 reveals that the number of defects per unit (DPU) will be constant as long as the following expression is equal to a constant $(\mathbf{C})$ :

$$
\mathrm{C}=\mathrm{TM}-\boldsymbol{t}_{0} \cdot \mathrm{N}_{\mathrm{a}}
$$

From Equation 3, lines of constant defects per unit (iso-DPU) can be plotted on the same figure with the Design for Assembly data for the alternative concepts as shown in Figure 8. The slope of the lines of constant defects per unit (iso-DPU) is equal to the threshold assembly time $t_{0}$. Note that the relative potential defect rate can be determined by the perpendicular distance above or below the iso-DPU line passing through the baseline concept. The defect rate decreases as distance below this line increases.

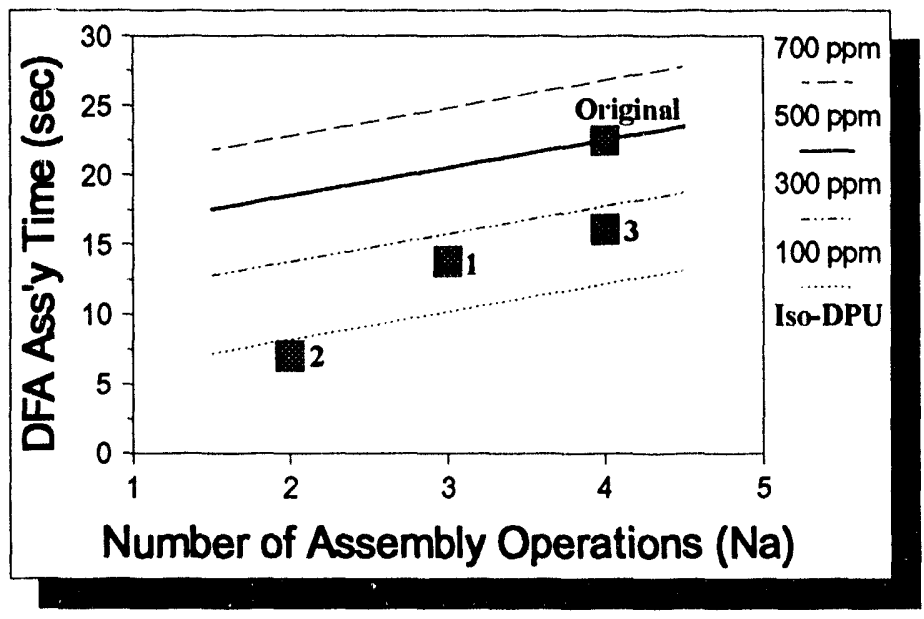

Figure 8. Predicted Design for Assembly (DFA) time versus the number of assembly operations for four products from Figure 7. The solid line is for constant defects per unit equal to the baseline concept labeled "Original" (based on $t_{0}=2 \mathrm{sec} / \mathrm{op}$ ). Numbers indicate other concepts.

Concept 3 with the pin lock cover is not ideal from the standpoint of ease of assembly because the pins are not inserted in a top-down manner. However, this product illustrates a very important point. Comparing Concepts 1 and 3, the projected defect rates are similar in spite of the fact that Concept 3 requires more assembly time and more assembly operations! This relationship at first seems counter-intuitive, but results from the inverse relationship of defect rates with the number of assembly operations from Equation 1. In addition to the data presented earlier, one automotive manufacturer provided defect data spanning billions of assembly operations. Although we could not evaluate the data in the same manner as the Motorola and disk drive data because the automotive manufacturer did not have Design for Assembly evaluations, one important and consistent trend was the negative correlation with the number of assembly operations.

Comparing the results of Table 1 and Figure 8, we can see that the relative conformance quality of the conct.pts studied can be predicted by the graphical method without the need for lengthy calculations. This comparison is based on the assumption that the same 
criteria, like 6 sigma, is used to control the level of variation of all concepts. A line of the appropriate slope can simply be drawn through the point representing the baseline concept and the relative distance above or below the line can be used to estimate the relative potential defect rates, providing valuable input during concept comparison and selection with a minimum amount of analysis.

Because the level of quality control within an organization can change, the defect rates predicted using this method should not be viewed as either absolute or unalterable. The great advantage of this method is that it provides a useful relative estimate of the defect rates of alternate concepts that is not sensitive to changes in the level of quality control.

\section{Conclusions}

Achieving defect rates in the range of 1-10 parts per million, the new world class standard, requires a clear understanding of the cause of defects. We have shown that errors and variation are distinctly different defect sources. In contrast to the dispersion observed in every naturally occurring process, errors, such as omitting a part during assembly, can only be described in terms of probability. Errors in the production environment are such extremely rare events, that they are poorly characterized using traditional statistical methods based on small samples where outliers are often discarded. Although errors rarely occur, there are so many different types of error that error is often a major source of defects in products.

This reveals a critical limitation of statistical methods in achieving the highest levels of conformance quality. Our assessment of the distinction between error and variation is substantiated by experience at Motorola which has shown that extremely low defect rates were only achieved when methods for eliminating error were used in conjunction with the control of variation.

The distinct difference between error and variation suggests that a comprehensive or global model of product defect rates cannot be based on either of these concepts independently. Reducing product complexity eliminates opportunities for error and decreases the probability of defects resulting from variation. Thus, the link between complexity and defects is a more global or comprehensive model useful in predicting defects. However, industry-wide studies have shown poor correlations between defect rates and measures of complexity due to large differences in the level of quality control between manufacturers.

Using Design for Assembly methods to measure product complexity we have shown that defect rates are highly correlated with complexity within individual organizations. The identified link between complexity and defects provides a new means of benchmarking the relative quality control of organizations producing dissimilar products and aids in identifying important opportunities for reducing defects. Using the relationship between defects and complexity identified in this study, the potential defect rates of product 
concepts can be compared in the earliest stages of product develnpment with minimum effort, providing a powerful new tool useful in product development.

The concepts presented in this study have a potentially profound impact on the understanding of defects and the opportunities for improvement. It opens the way for the development of a quality strategy which can identify the most efficient method of reducing defects for each organization depending on their relative strengths and weaknesses in the control of error, variation, and product complexity. 


\section{REFERENCES}

The Associated Press, "Space shuttle workers afraid to report errors, study finds". The Modesto Bee, Modesto, July 27, 1993.

Barkan, P., \& C. M. Hinckley, "The Benefits and Limitations of Structured Methodologies in Product Design". Manufacturing Review, Vol. 6, No. 3, September, 1993, pp. 211-219.

Boothroyd, G., Dewhurst, P. (1985). Product Design for Assembly. Boothroyd Dewhurst, Inc., Section 2, Kingston, 1985.

Brannan, B. (1991). "Six Sigma Quality and DFA-DFMA Case Study/Motorola Inc". Boothroyd \& Dewhurst DFM Insight Vol. 2, Winter, pg 1-3.

Buzzell, R. D., and B. T. Gale (1987). The PIMS (Profit Impact of Market Strategy) PrinciplesLinking Strategy to Performance. Chapter 6, pp. 103-131.

Card, S., W. English, and B. Burr (1978). "Evaluation of mouse, rate-controlled isometric joystick, step keys and text keys for test selection on a CRT". Ergonomics, 21(8), pp. 601-613.

Ekings, J. D. (1988), "Assembly Industries". Juran's Quality Control Handbook, Section 30, J. M. Juran, editor in chief, Frank M. Gryna associate editor, Fourth Edition, McGraw-Hill, New York, 1988., pp. 24.6.

Gatchsll, S. M. (1979). "The Effect of Part Proliferation on Assembly Line Operators' Decision Making Capabilities". Proceedings of the Human Factors Society, 23rd Annual Meeting, (Santa Monica : The Human Factors Society).

Gebala, D. (1992), Correspondence, Motorola, Inc., Advanced Manufacturing Technologies, Fort Lauderdale, FL, August 7, 1992.

Gryna, F. M. (1988). "Manufacturing Planning". Chapter 16, Juran's Quality Control Handbook, J. M. Juran, editor in chief, Frank M. Gryna associate editor, Fourth Edition, McGraw-Hill, New York, pp. 16.19-16.21.

Harris, D. H. (1966). "The Effect of Equipment Complexity on Inspection Performance". Journal of Applied Psychology 50, 236-237.

Harris, D. H., and F. B. Chaney (1969). Human Factors in Quality Assurance. Wiley, New York, p. 9.

Harry, M. J. and R. Stewart (1988), "Six Sigma Mechanical Design Tolerancing". Motorola Publication No. $6 \sigma-2-10 / 88$, Scottsdale, AZ.

Heggem, R. (April 30, 1992), "Proactive Tolerance Analysis". Applied Computer solutions, Santa Clara.

Held, D. O. (June 1993), "Quality Advisor". Manufacturing Engineering, pp.12.

Hinckley, C. M. (1993). A Global Conformance Quality Model - A New Strategic Tool for Minimizing Defects Caused by Variation, Error, and Complexity. A Dissertation submitted to the Department of Mechanical Engineering and the Committee of Graduate Studies of Stanford University, Stanford.

Ishikawa, K. (1990). Guide to Quality Control. Asian Productivity Organization, Quality Resources, White Plains, Seventh Printing.

Jerison, H. J. (1963). "On the Decrement Function in Human Vigilance". In D. N. Buckner and J. J. McGrath (Eds.), Vigilance: A symposium, New York, McGraw-Hill, pp. 199-216.

Lafferty, J. P., (October 1992) "Quality Advisor," Manufacturing Engineering.

MacKenzie, I. S., A. Sellen, and W. Buxton (1991). "A Comparison of Input Devices in Elemental Pointing and Dragging Tasks". Human Factors in Computing Systems-Reaching Through Technology, CHI 1991 Conference Proceedings, Edited by S. P. Robertson, G. M. Olson, and J. S. Olson, Addison Wesley Publishing Co., Reading, pp. 161-166. 
Meister, D. (1961). Analysis of Human Initiated Equipment Failures During Category I Testing. OSTF-1, Report REL R-54. General Dynamics/Astronautics, San Diego.

Nikkan Kogyo Shimbun, Ltd./Factory Magazine, Ed. (1988). Poka-yoke-Improving Product Quality by Preventing Defects. Edited by Productivity Press, Cambridge.

Oberg, E., F. D. Jones, and H. L. Horton (1984). Machinery's Handbook - 22nd Edition. Edited by H. H. Ryffel and J. H. Geronimo, Industrial Press Inc., New York, p. 1532.

Olivera, R. (1988). "6 Sigma/Fit Tolerance Analysis". Communications Sector, Motorola Inc., Schaumburg.

Park, K. S. (1987), Human Reliability - Analysis, Prediction, and Prevention of Human Errors, Elsevier, Amsterdam, p. 197.

Rasmussen, J. (1981). Human Errors, A taxonomy for describing human malfunctions in industrial intallations. Report No. RIS $\emptyset-M-2304$, Roskilde, Denmark: RIS $\emptyset$ National Laboratories.

Rasmussen, J. (1985), "Trends in Human Reliability Analysis". Ergonomics, 28(8), pp. 11851195.

Research Needs and Technological Opportunities in Mechanical Tolerancing (1988). Results of an International Workshop, Select Panel on Research Opportunities in Mechanical Tolerancing held in Orlando Florida, Sep 28-Oct 2, for the US National Science Foundation Design and Manufacturing Systems Division, ASME CRTD-15, New York.

Rook, L. W., Jr. (1962). Reduction of Human Error in Production. SCTM 93-62(14), Sandia National Laboratories, Division 1443, June 1962.

Rook, L. W., Jr. (1965). Motivation and Human Error. SC-TM-135, Sandia National Laboratories, Albuquerque, p. 5.

Ross, P. J. (1988), Taguchi Techniques for Quality Engineering. McGraw-Hill, New York.

Sanger, D. E. (November 1, 1990), "U.S. Suppliers Get a Toyota Lecture". The New York Times.

Shingo, S. (1986). Zero Quality Control: Source Inspection and the Poka-yoke System. Translated by Andrew P. Dillon, Productivity Press, Cambridge.

Smith, B. (1993), "Making a War on Defects-Six Sigma Design". IEEE Spectrum, September, 1993, pp. 43-47.

Stalk, G., Jr., and T. M. Hout (1990). Competing Against Time-How Time-Based Competition is Reshaping Global Markets. The Free Press.

Swain, A. D. and H. E. Guttmann (1983), Handbook of Human Reliability Analysis with Emphasis on Nuclear Power Plant Applications, NUREG/CR-1278, SAND80-0200, RX, AN, Sandia National Laboratories, Albuquerque, August, 1983.

Tsuda, Y. (1993), "Implications of Fool Proofing in the Manufacturing Process". Quality Through Engineering Design, Edited by W. Kuo, Elsevier, pp. 79-95.

Voegtlen, H. D. (1988). "Complex Industries". Section 31., Juran's Quality Control Handbook, J. M. Juran, editor in chief, Frank M. Gryna associate editor, Fourth Edition, McGraw-Hill, New York, pp. 31.1-31.24.

Womack, J. P., D. T. Jones \& D. Roos (1990). The Machine that Changed the World. Rawson Associates, New York, p. 93, 96. 


\section{UNLIMITED RELEASE}

\section{INITIAL DISTRIBUTION}

\section{Susan Held}

Co-Program Manager, Leadership Through Quality Staff Albuquerque Operations Office

U. S. Department of Energy

P. O. Box 5400

Albuquerque, NM 87185-5400

Mark J. Kahnke

Director, Total Quality Management and Planning

AlliedSignal Inc., Kansas City Division, Mail Post 2A44

P.O. Box 419159

Kansas City, Mo. 64141-6159

Don E. Michel

President and General Manager

EG\&G Mound Applied Technologies, Inc.

P.O. Box 3000

Miamisburg, $\mathrm{OH} 45342-3000$

Melinda K. Bynum

TQM Director

Rocky Flats Plant

P.O. Box 464

Golden, CO 80402-0464

Anders W. Lundberg

Special Assistant for Weapons Safety Studies

Defense Technologies Engineering Division

Lawrence Livermore National Laboratories

P. O. Box 808, L- 125

Livermore, CA 94551

Harry Flaugh

WX Deputy Division Leader

Los Alamos National Laboratories

WX-DO, MS P945

Los Alamos, NM 87545

Susan H. Alexander

Manager, Organizational Improvement Programs

Martin Marrietta Energy Systems, Inc.

Y-12 Site, Building 9119, Mail Stop 8236

Oak Ridge, TN 37831 
William $F$. Ierna

Director-Quality Assurance

Martin Marietta Specialty Components

P.O. Box 2908

Largo, FL 34649

J. C. Drummond

Division Manager, Quality

Mason \& Hanger -Silas Mason Co., Inc.

Building 12-28

P.O. Box 30020

Amarillo, TX 79177

Dennis L. Hayes

Manager, Tritium Program Coordinator

Westinghouse Savannah River Site

235-H

P.O. Box 516

Aiken, SC 29802

MSTP

0112 P. M. Stanford, 10000

0141 M. R. Kestenbaum, 11000

0149 C. P. Robinson, 4000

0151 G. Yonas, 9000

0185 K. G. Haug, 10100

0186 C. E. Emery, 3000

0360 A. R. C. Westwood, 1000

0366 R. T. Johnson, 1040

0429 W. C. Nickell, 5100

0455 G. R. Otey, 4100

0463 R. L. Hagengruber, 5000

0513 H. W. Schmitt, 2000

0517 M. Prickett, 2508

0724 D. L. Hartley, 6000

0872 A. Beradino, 5408

0953 W. E. Alzheimer, 2900

1067 M. L. Jones, 7000

1357 A. A. Trujillo, 12913

1357 T. Olascoaga, 12914

1359 D. W. Bushmire, 12911

1361 M. R. Baca, 12909

1363 B. Hawkinson, 12662

1365 J. K. Gabaldon, 12903

1369 C. M. Tapp, 12900 
R. R. Prairie, 12908

1380

W. D. Siemens, 4200

9001

J. C. Crawford, 8000

Attn: M. E. John, 8100

W. J. McLean, 8300

P. N. Smith, 8500

R. C. Wayne, 8700

9005

J. B. Wright, 5300

9006

9007

E. E. Ives, 5200

9014

T. M. Dyer, 8800

9036

D. R. Henson, 5371

9037

C. T. Yokomizo, 8007

9103

R. J. Detry, 8200

9103

G. Thomas, 8111

9105

P. R. Bryson, 8111

9203

L. A. Hiles, 8400

E. T. Cull, 5354

9405

R. E. Stoltz, 8008

9408

A. J. West, 8414

9409

L. N. Tallerico, 8205

9901

L. A. West, 8600

9901

C. M. Hinckley, 8603

9021 Publications for OSTI(10), 8535

9021 Publications/Technical Library Processes, 8535

0899

Technical Library Processes Department (4), 7141

9018 Central Technical Files (3), 8523 


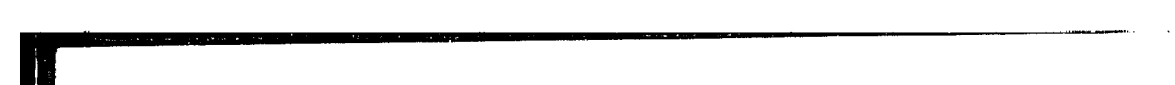

